Copyright (C) 2005 IEEE. Reprinted from

IEEE Transactions on Information Theory, 2005; 51 (5):1731-1738

This material is posted here with permission of the IEEE. Such permission of the IEEE does not in any way imply IEEE endorsement of any of the University of Adelaide's products or services. Internal or personal use of this material is permitted. However, permission to reprint/republish this material for advertising or promotional purposes or for creating new collective works for resale or redistribution must be obtained from the IEEE by writing to pubs-permissions@ieee.org.

By choosing to view this document, you agree to all provisions of the copyright laws protecting it. 


\title{
Risk-Sensitive Filtering and Smoothing for Continuous-Time Markov Processes
}

\author{
W. Paul Malcolm, Robert J. Elliott, and Matthew R. James, Fellow, IEEE
}

\begin{abstract}
We consider risk sensitive filtering and smoothing for a dynamical system whose output is a vector process in $\mathbb{R}^{2}$. The components of the observation process are a Markov process observed through a Brownian motion and a Markov process observed through a Poisson process. Risk-sensitive filters for the robust estimation of an indirectly observed Markov state processes are given. These filters are stochastic partial differential equations for which robust discretizations are obtained. Computer simulations are given which demonstrate the benefits of risk sensitive filtering.
\end{abstract}

Index Terms-Change of measure, martingales, risk-sensitive filtering.

\section{INTRODUCTION}

I $\mathrm{N}$ this paper, we consider robust nonlinear filtering and smoothing in the presence of model uncertainties. To derive filters robust to model uncertainties, we use the so-called risk-sensitive criterion [1]. The dynamical models considered involve indirect observations of a continuous-time Markov process. For a general model, we consider a vector observation process, where the two components of this vector are different observations of the same Markov state process. The two observation processes are: i) the Markov process observed through a Brownian motion, ii) the Markov process observed through a Poisson process. This particular model has also been considered in [2].

The paper is organized as follows. In Section II, the signal models for the state process and the observation process are defined. In Section III, we give a definition of risk-sensitive filtering and present risk-sensitive filters for our stochastic model. In this section, we derive versions of our risk-sensitive filters which are robust to time discretization. We obtain these versions by adapting the transformation techniques introduced by Clark [3]. In Section IV, we provide a corresponding robust smoothing algorithm. Finally, in Section VI, we present a simulation study

Manuscript received September 13, 2000; revised October 25, 2004. The work of W. P. Malcolm was supported by the National ICT Australia. The National ICT Australia is funded by the Australian Government's Department of Communications, Information Technology and the Arts and the Australian Research Council through Backing Australia's Ability and the ICT Centre of Excellence program.

W. P. Malcolm is with the National ICT Australia (NICTA), Locked Bag 8001, Canberra ACT 2601 Australia (e-mail: paul.malcolm@nicta.com.au).

R. J. Elliott is with the Haskayne School of Business, Scurfield Hall, University of Calgary, Calgary, AB T2N 1N4, Canada (e-mail: relliott@ucalgary.ca).

M. R. James is with the Faculty of Engineering and Information Technology, Department of Engineering, the Australian National University, Canberra ACT 0200, Australia (e-mail: matthew.james@ anu.edu.au).

Communicated by J. A. O'Sullivan, Associate Editor for Detection and Estimation.

Digital Object Identifier 10.1109/TIT.2005.846405 to show the performance benefits in a counting process observation scenario.

\section{SignAl Models}

In this section, we describe dynamics for an indirectly observed state process and dynamics for two scalar-valued observation processes, each influenced by the same state process. All signal models are defined on the fixed probability space $(\Omega, \mathcal{F}, P)$.

\section{A. State Process}

For our state variable, consider a Markov process in continuous time whose state space is a countable finite set. Our representation for this process follows that used in [4], whereby one can, without loss of generality, take the state space to be the set $\mathcal{L} \triangleq\left\{\boldsymbol{e}_{1}, \boldsymbol{e}_{2}, \ldots, \boldsymbol{e}_{n}\right\}$, whose elements $\boldsymbol{e}_{i}$ are column vectors in $\mathbb{R}^{n} ; \boldsymbol{e}_{i}$ has unity in the $i$ th position and zero elsewhere. The essential point of this representation is the state process dynamics can then be written down in a semimartingale form (see [4])

$$
X_{t}=X_{0}+\int_{0}^{t} A X_{u} d u+M_{t} .
$$

Here $M$ is a martingale with respect to the sigma field generated by the process $X$ and $A$ is an $n \times n$ rate matrix for $X$. Suppose that our Markov chain in fact takes real values $\lambda^{1}, \ldots, \lambda^{n}$. Write $\lambda \triangleq\left(\lambda^{1}, \ldots, \lambda^{n}\right)^{\prime}$. Then with the underlying Markov chain $X$ taking values in $\mathcal{L}$, the related real-valued Markov process is just $\langle X, \boldsymbol{\lambda}\rangle$. We wish to consider the case where $\left\langle\boldsymbol{\lambda}, \boldsymbol{e}_{i}\right\rangle$ are possible rate parameters of a Poisson process.

\section{B. Observation Processes}

We suppose that the Markov process $X$ of Section II-A is not directly observed; instead, there are two scalar-valued observation processes $y$ and $N$. The vector-valued observation process $Y=\left\{Y_{t}, 0 \leq t\right\}$ is a process taking values in $\mathbb{R}^{2}$, where

$$
Y \triangleq\left[\begin{array}{c}
y \\
N
\end{array}\right] \text {. }
$$

Case 1, the $y$-Component of $Y$ : The process $y$ is an observation of $X$ through a Brownian motion, with dynamics

$$
y_{t}=\int_{0}^{t}\left\langle X_{u}, g\right\rangle d u+w_{t} .
$$

Here, $\boldsymbol{g} \triangleq\left(g^{1}, g^{2}, \ldots, g^{n}\right)^{\prime}$ is a vector of the drift coefficients and $w_{t}$ is a standard Brownian motion, independent of the process $X$. 
Case 2, the $N$-Component of $Y$ : The process $N$ is a Markov modulated Poisson process whose intensity process depends upon the process $X$. Its dynamics are

$$
N_{t}=\int_{0}^{t}\left\langle X_{u}, \lambda\right\rangle d u+L_{t} .
$$

Here $L$ is a $\left(\mathcal{G}_{t}=\sigma\left\{Y_{u}, X_{u} ; u \leq t\right\}, P\right)$-martingale.

The two main problems we consider in this paper are; the robust estimation of the filter probabilities $P\left(X_{t}=e_{i} \mid \mathcal{Y}_{t}\right)$, for $i=1,2, \ldots, n$ and the robust estimation of the smoother probabilities $P\left(X_{t}=e_{i} \mid \mathcal{Y}_{T}\right)$, for $t \leq T$ and $i=1,2, \ldots, n$. Here the term robust is taken in the risk sensitive sense [1], [5].

\section{Reference Probability}

A reference probability $P^{\dagger}$ is introduced under which the two components of the observation process $Y_{t}$ are, respectively, a standard Brownian motion and a standard Poisson process. That is, under the measure $P^{\dagger}$, the dynamics have the form

$$
P^{\dagger}\left\{\begin{array}{l}
d X_{t}=A X_{t} d t+d M_{t} \\
d Y_{t}=\left[\begin{array}{l}
d w_{t} \\
d N_{t}
\end{array}\right]
\end{array}\right.
$$

Of course, under the $P$, the dynamics have the form

$$
P\left\{\begin{array}{l}
d X_{t}=A X_{t} d t+d M_{t} \\
d Y_{t}=\left[\begin{array}{l}
\left\langle X_{t}, \boldsymbol{g}\right\rangle d t+d w_{t} \\
\left\langle X_{t}, \boldsymbol{\lambda}\right\rangle d t+d m_{t}
\end{array}\right] .
\end{array}\right.
$$

\section{RISK-SENSITIVE FILTERING}

\section{A. Risk-Sensitive Estimation}

Risk-sensitive filtering provides a type of robust filtering. The term "robust" is a relative one with a variety of possible meanings. For example, an estimation scheme could be robust against assumptions of independence, or robust against the parameters of a certain distribution. One general definition of robustness is "robustness signifies insensitivity against small deviations from assumptions," [6]. In this paper, we consider robustness against uncertainties in the parameters of a probabilistic model for a stochastic dynamical system. We assume that there always exists a true and fixed, yet possibly unknown, probabilistic model for the system of interest. We denote the distribution of this true model by $P_{0}$. Since we declare that the model being used may not necessarily correspond to the true model, we denote the design model by $P_{d}$, where $d$ is a label corresponding to the design probability model.

Suppose we are interested in the estimation of a scalar-valued function of the Markov process $X$; let this function be denoted by $\xi$. Further, suppose that the function $\xi(\cdot)$ is $\mathcal{Y}_{t}$-measurable.

Definition 1: Suppose $c$ and $h$ are convex scalar "cost" functions with $c(\xi, \hat{\xi})=0$ and $h(\xi, \hat{\xi})=0$, iff $\xi=\widehat{\xi}$. For example, $c(\xi, \hat{\xi})=\|\xi-\hat{\xi}\|^{2}$. Suppose further that there exists a process $\left\{\hat{\xi}_{t}^{\mu}, 0 \leq t\right\}$ which is continuous on the right with limits on the left (that is, CORLOL) and which satisfies

$$
\widehat{\xi}_{t}^{\mu}=\underset{\widehat{\xi} \in \mathcal{Y}_{t}}{\operatorname{argmin}} E_{d}\left[\exp \mu\left\{\int_{0}^{t} c\left(\xi_{u}, \widehat{\xi}_{u}\right) d u+h\left(\xi_{t}, \widehat{\xi}_{t}\right)\right\}\right] .
$$

Then $\widehat{\xi}_{t}^{\mu}$ is a risk sensitive estimate of $\xi_{t}$.
Notation: The expectation $E_{d}[\cdot]$ in (3.7) denotes expectation under the design distribution $P_{d}$, and the parameter $\mu>0$ is the so-called risk-sensitive parameter. Quantitatively, the risksensitive parameter determines the degree of "risk."

Risk-sensitive estimators defined by (3.7) enjoy an estimation error which is upper-bounded. This is in stark contrast to estimators defined by the minimum mean-square error criterion for which no such bound exists. The upper error bound corresponding to (3.7) is determined in [1] and is

$$
\mathbf{E}_{0}\left[\int_{0}^{t} c\left(\xi_{u}, \widehat{\xi}_{u}^{\mu}\right) d u\right] \leq \frac{\left[\log J_{t}\left(\widehat{\xi}^{\mu}\right)+R_{t}\left(P_{0} \mid P_{d}\right)\right]}{\mu} .
$$

Here

$$
J_{t}\left(\widehat{\xi}^{\mu}\right)=\mathbf{E}_{d}\left[\exp \mu\left\{\int_{0}^{t} c\left(\xi_{u}, \widehat{\xi}_{u}^{\mu}\right) d u+h\left(\xi_{t}, \widehat{\xi}_{t}^{\mu}\right)\right\}\right]
$$

and $R_{t}\left(P_{0} \mid P_{d}\right)$ denotes the relative entropy between the two probability models $P_{0}$ and $P_{d}$. This result (due to Boel et al. [1]) is an important theoretical contribution, as it established a "precise" meaning for risk-sensitive estimation. The bound defined by (3.8) tells us that the error in risk-sensitive estimation has an upper bound given by the sum of two terms. The first term coincides with a cost if the model were known precisely, while the second term is a measure of distance between the true probability and the design probability models for the system.

\section{B. Filtering Equations}

Suppose $c(\cdot)$ is a cost function, as in Definition 1 and

$$
Z_{t} \triangleq \operatorname{diag}\left\{z_{t}\right\},
$$

where

$$
z_{t} \triangleq \exp \left(\mu \int_{0}^{t} c\left(\xi_{u}, \widehat{\xi}_{u}^{\mu}\right) d u\right) .
$$

Consider the state variable $X_{t}$ with state space $\mathcal{L}$.

The risk-sensitive unnormalized measure of $X_{t}$, given the history $\mathcal{Y}_{t}$, is defined by

$$
q_{t}^{\mu} \triangleq E^{\dagger}\left[X_{t} \Lambda_{t} Z_{t} \mid \mathcal{Y}_{t}\right] .
$$

The expectation in this definition is with respect to the reference measure $P^{\dagger}$ and $\Lambda_{t}$ denotes the Radon-Nikodym derivative

$$
\Lambda_{t}=\left.\frac{d P}{d P^{\dagger}}\right|_{\mathcal{G}_{t}} .
$$

Write

$$
c_{i}\left(\widehat{\xi}_{t}^{\mu}\right) \triangleq c\left(\boldsymbol{e}_{i}, \widehat{\xi}_{t}^{\mu}\right) .
$$

Theorem 1: The process $q^{\mu}$ satisfies the stochastic integral equation

$$
\begin{aligned}
q_{t}^{\mu}= & q_{0}^{\mu}+\int_{0}^{t}\left[A+\mu \times \operatorname{diag}\left\{c_{i}\left(\widehat{\xi}_{u}^{\mu}\right)\right\}\right] q_{u}^{\mu} d u \\
& +\int_{0}^{t} \operatorname{diag}\left\{\left\langle\boldsymbol{g}, \boldsymbol{e}_{i}\right\rangle\right\} q_{u}^{\mu} d y_{u} \\
& +\int_{0}^{t} \operatorname{diag}\left\{\left\langle\lambda, \boldsymbol{e}_{i}\right\rangle-1\right\} q_{u-}^{\mu}\left(d N_{u}-d u\right) .
\end{aligned}
$$

A proof of Theorem 1 is given in the Appendix. 
Corollary 1: The process $q^{\mu}$, defined by (3.14), is an unnormalized measure-valued process. The corresponding normalized measure-valued process $\Pi^{\mu}=\frac{q^{\mu}}{\left\langle q^{\mu}, \mathbf{1}\right\rangle}$ has dynamics

$$
\begin{aligned}
\Pi_{t}^{\mu}= & \Pi_{0}^{\mu}+\int_{0}^{t}\left[A+\mu \times \operatorname{diag}\left\{c_{i}\left(\widehat{\xi}_{u}^{\mu}\right)\right\}\right] \Pi_{u}^{\mu} d u \\
& \left.+\int_{0}^{t} \operatorname{diag}\left\{\left\langle\boldsymbol{g}, \boldsymbol{e}_{i}\right\rangle\right\} \Pi_{u}^{\mu}-\Pi_{u-}^{\mu}\left\langle\Pi_{u}^{0}, \boldsymbol{g}\right\rangle\right\} d \nu_{u}^{(1)} \\
& +\int_{0}^{t}\left\{\frac{\operatorname{diag}\left\{\left\langle\boldsymbol{\lambda}, \boldsymbol{e}_{i}\right\}\right\rangle \Pi_{u-}^{\mu}-\Pi_{u-}^{\mu},\left\langle\Pi_{u}^{0}, \boldsymbol{\lambda}\right\rangle}{\left\langle\Pi_{u}^{0}, \boldsymbol{\lambda}\right\rangle}\right\} d \nu_{u}^{(2)} .
\end{aligned}
$$

Here

$$
\begin{aligned}
& d \nu_{t}^{(1)}=d y_{t}-\left\langle\Pi_{t}^{0}, \boldsymbol{g}\right\rangle d t \\
& d \nu_{t}^{(2)}=d N_{t}-\left\langle\Pi_{t}^{0}, \boldsymbol{\lambda}\right\rangle d t
\end{aligned}
$$

and $\Pi_{t}^{0}$ denotes risk neutral expectation, for example, $\left\langle\Pi_{t}^{0}, \boldsymbol{g}\right\rangle=$ $E\left[\left\langle X_{t}, \boldsymbol{g}\right\rangle \mid \mathcal{Y}_{t}\right]$.

A proof of Corollary 1 is given in the Appendix.

Remark 1: The corresponding risk-neutral form of (3.14), and (3.15), are recovered by considering the limit, $\lim _{\mu \rightarrow 0} \frac{J_{t}\left(\hat{(}_{t}^{\mu}\right)}{\mu}$.

\section{State Estimation}

In the previous subsection, we defined a stochastic integral equation satisfied by the conditional distribution $q_{t}^{\mu}$. We now use this distribution to write down a risk-sensitive state estimator for the function $\xi_{t}$. In Definition 1, the risk-sensitive estimate $\widehat{\xi}_{t}^{\mu}$ was defined as an estimate which minimized an expectation involving an exponential cost. It was shown in [1] that one can identify a version of the minimum risk-sensitive estimator for $\xi_{t}$ which attains the minimum defined by (3.7). The equation for the risk-sensitive estimate $\hat{\xi}_{t}^{\mu}$ is

$$
\widehat{\xi}_{t}^{\mu} \triangleq \underset{\widehat{\xi} \in \mathbb{R}}{\operatorname{argmin}} \sum_{i=1}^{n} \exp \left(\mu \times c\left(\xi^{i}, \widehat{\xi}\right)\right) \frac{q_{t}^{\mu}}{\left\langle q_{t}^{\mu}, \mathbf{1}\right\rangle}
$$

where 1 denotes the vector in $\mathbb{R}^{n}$ whose elements are each unity. Taken together, the stochastic differential (3.14) and the state estimator (3.18) comprise a risk-sensitive filter. To implement such a filter, one needs to first compute the density $q_{t}^{\mu}$ at time $t$, then, using this density, determine the estimate $\hat{\xi}_{t}^{\mu}$ defined by (3.18).

\section{Transformed Filtering Equations}

Notation: Let $\phi_{t}^{i}$ be a scalar-valued stochastic process defined by

$$
\phi_{t}^{i} \triangleq \exp \left(\left\langle\boldsymbol{g}, \boldsymbol{e}_{i}\right\rangle y_{t}-\frac{1}{2}\left|\left\langle\boldsymbol{g}, \boldsymbol{e}_{i}\right\rangle\right|^{2} t\right) .
$$

Let $\Phi_{t}$ be a matrix exponential, defined by

$$
\Phi_{t} \triangleq \operatorname{diag}\left\{\phi_{t}^{1}, \phi_{t}^{2}, \ldots, \phi_{t}^{n}\right\} .
$$

Notation: Let $\gamma_{t}^{i}$ be a scalar-valued stochastic process defined by

$$
\gamma_{t}^{i} \triangleq \exp \left(\left(1-\left\langle\lambda, \boldsymbol{e}_{i}\right\rangle\right) t\right)\left\langle\boldsymbol{\lambda}, \boldsymbol{e}_{i}\right\rangle^{N_{t}}
$$

where $\left\langle\boldsymbol{\lambda}, \boldsymbol{e}_{i}\right\rangle$ is the $i$ th Poisson rate and $N_{t}$ is the observation process defined by the model (2.4). Let $\Gamma_{t}$ be a matrix exponential defined by

$$
\Gamma_{t} \triangleq \operatorname{diag}\left\{\gamma_{t}^{1}, \gamma_{t}^{2}, \ldots, \gamma_{t}^{n}\right\}
$$

Equation (3.14) can be solved by variation of constants. Consider the two subsidiary equations for the diagonal matrices $\Phi_{t}$ and $\Gamma_{t}$

$$
\begin{aligned}
d \Phi_{t} & =\operatorname{diag}\left\{\left\langle\boldsymbol{g}, \boldsymbol{e}_{i}\right\rangle\right\} \Phi_{t} d y_{t}, & & \Phi_{0}=I \\
d \Gamma_{t} & =\operatorname{diag}\left\{\left\langle\boldsymbol{\lambda}, \boldsymbol{e}_{i}\right\rangle-1\right\} \Gamma_{t}\left(d N_{t}-d t\right), & & \Gamma_{0}=I
\end{aligned}
$$

where $I$ is the identity matrix in $\mathbb{R}^{n}$. The matrices $\Phi_{t}$ and $\Gamma_{t}$ are diagonal so we are considering the following scalar equations for each diagonal entry:

$$
\begin{array}{rlrl}
d \phi_{t}^{i} & =\left\langle\boldsymbol{g}, \boldsymbol{e}_{i}\right\rangle \phi_{t}^{i} d y_{t}, & & \phi_{0}^{(i)}=1 \\
d \gamma_{t}^{i}=\left(\left\langle\boldsymbol{\lambda}, \boldsymbol{e}_{i}\right\rangle-1\right) \gamma_{t}^{i}\left(d N_{t}-d t\right), & \gamma_{0}^{(i)}=1 .
\end{array}
$$

Therefore,

$$
\begin{aligned}
& \phi_{t}^{i}=\exp \left\{\left\langle\boldsymbol{g}, \boldsymbol{e}_{i}\right\rangle y_{t}-\frac{1}{2}\left\langle\boldsymbol{g}, \boldsymbol{e}_{i}\right\rangle^{2} t\right\} \\
& \gamma_{t}^{i}=\left(\left(\left\langle\boldsymbol{\lambda}, \boldsymbol{e}_{i}\right\rangle-1\right) t\right)\left\langle\boldsymbol{\lambda}, \boldsymbol{e}_{i}\right\rangle^{N_{t}} .
\end{aligned}
$$

Consequently, $\Phi_{t}$ and $\Gamma_{t}$ are nonsingular and

$$
\begin{aligned}
& \Phi_{t}^{-1}=\operatorname{diag}\left\{1 /\left(\phi_{t}^{i}\right)\right\} \\
& \Gamma_{t}^{-1}=\operatorname{diag}\left\{1 /\left(\gamma_{t}^{i}\right)\right\} .
\end{aligned}
$$

Consider the solution of $\bar{q}_{t}^{\mu}$ of the ordinary differential equation

$$
\frac{d \bar{q}_{t}^{\mu}}{d t}=\Phi_{t}^{-1} \Gamma_{t}^{-1}\left[A+\mu \times \operatorname{diag}\left\{c_{i}\left(\widehat{\xi}_{t}^{\mu}\right)\right\}\right] \Phi_{t} \Gamma_{t} \bar{q}_{t}^{\mu}
$$

with initial condition $\bar{q}_{0}^{\mu}=q_{0}^{\mu}$.

Theorem 2: The process $q^{\mu} \triangleq \Phi \Gamma \bar{q}^{\mu}$ is a unique solution of the stochastic integral equation

$$
\begin{aligned}
q_{t}^{\mu}=q_{0}^{\mu}+\int_{0}^{t} & {\left[A+\mu \times \operatorname{diag}\left\{c_{i}\left(\hat{\xi}_{u}^{\mu}\right)\right\}\right] q_{u}^{\mu} d u } \\
& +\int_{0}^{t} \operatorname{diag}\left\{\left\langle\boldsymbol{g}, \boldsymbol{e}_{i}\right\rangle\right\} q_{u-}^{\mu} d y_{u} \\
& +\int_{0}^{t} \operatorname{diag}\left\{\left\langle\boldsymbol{\lambda}, \boldsymbol{e}_{i}\right\rangle-1\right\} q_{u-}^{\mu}\left(d N_{u}-d u\right) .
\end{aligned}
$$

Proof of Theorem 2: Note that the matrices $\Phi_{t}, \Phi_{t}^{-1}, \Gamma_{t}$, and $\Gamma_{t}^{-1}$ are all diagonal and, therefore, commute. Setting $\widetilde{q}_{t}^{\mu} \triangleq$ $\Phi_{t} \Gamma_{t} \bar{q}_{t}^{\mu}$, we see that

$$
\begin{aligned}
d \widetilde{q}_{t}^{\mu}= & \Phi_{t} \Gamma_{t} d\left(\bar{q}_{t}^{\mu}\right)+d\left(\Phi_{t}\right) \Gamma_{t} \bar{q}_{t}^{\mu}+\Phi_{t} d\left(\Gamma_{t}\right) \bar{q}_{t}^{\mu} \\
= & \Phi_{t} \Gamma_{t} \Phi_{t}^{-1} \Gamma_{t}^{-1}\left[A+\mu \times \operatorname{diag}\left\{c_{i}\left(\widehat{\xi}_{t}\right)\right\}\right] \Phi_{t} \Gamma_{t} \bar{q}_{t}^{\mu} \\
& +\Phi_{t} \Gamma_{t} \operatorname{diag}\left\{\left\langle\boldsymbol{g}, \boldsymbol{e}_{i}\right\rangle\right\} \bar{q}_{t}^{\mu} d y_{t} \\
& +\Phi_{t} \Gamma_{t} \operatorname{diag}\left\{\left\langle\boldsymbol{\lambda}, \boldsymbol{e}_{i}\right\rangle-1\right\} \bar{q}_{t}^{\mu}\left(d N_{t}-d t\right) \\
= & {\left[A+\mu \times \operatorname{diag}\left\{c_{i}\left(\widehat{\xi}_{t}\right)\right\}\right] \widetilde{q}_{t}^{\mu} } \\
& +\operatorname{diag}\left\{\left\langle\boldsymbol{g}, \boldsymbol{e}_{i}\right\rangle\right\} \widetilde{q}_{t}^{\mu} d y_{t} \\
& +\operatorname{diag}\left\{\left\langle\boldsymbol{\lambda}, \boldsymbol{e}_{i}\right\rangle-1\right\} \widetilde{q}_{t}^{\mu}\left(d N_{t}-d t\right) .
\end{aligned}
$$

Therefore, $\widetilde{q}^{\mu}$ is a solution of (3.14). Since solutions of this equation are unique, the result follows.

The importance of Theorem 2 is that it identifies a process $\bar{q}^{\mu}$ which satisfies an ordinary differential equation. From this 
process, the process $q_{t}^{\mu}$ can always be determined. Further, to implement equations such as (3.14) on a digital computer, a discretization of continuous time must be used. Discretising (3.31) provides a robust discretization. Here the term "robust" refers to the sensitivity of $\bar{q}_{t}^{\mu}$ with respect to variations in the model parameters $\{A, \boldsymbol{g}, \lambda\}$.

Remark 2: The obvious special cases are obtained when either $\boldsymbol{\lambda}=\mathbf{0}$ or $\boldsymbol{g}=\mathbf{0}$.

\section{E. The Risk Sensitive Duncan-Mortensen-Zakai Equation}

We now write down a robust (to discretization) form of the Duncan-Mortensen-Zakai equation for the risk-sensitive filter of Theorem 2. The interesting point is that the observation process appears as a parameter, and to update the estimate at the next time point only the new observation is used.

From Theorem 2, the transformed process $\bar{q}^{\mu}$ satisfies the linear ordinary differential equation

$$
\frac{d \bar{q}_{t}^{\mu}}{d t}=\Phi_{t}^{-1} \Gamma_{t}^{-1}\left[A+\mu \times \operatorname{diag}\left\{c_{i}\left(\widehat{\xi}_{t}^{\mu}\right)\right\}\right] \Phi_{t} \Gamma_{t} \bar{q}_{t}^{\mu}
$$

To discretise this equation, we consider a regular partition in time

$$
0=t_{0}<t_{1}<\cdots<t_{k-1}<t_{k} \cdots
$$

where $t_{k}-t_{k-1}$ is a constant time step, denoted by $\Delta$. We make the following approximation of (3.34) between the sampling instants $t_{k-1}$ and $t_{k}$ :

$$
\begin{aligned}
\bar{q}_{t_{k}}^{\mu}= & \bar{q}_{t_{k-1}}^{\mu}+\int_{t_{k-1}}^{t_{k}} \Phi_{u}^{-1} \Gamma_{u}^{-1} \\
& \times\left\{A+\mu \times \operatorname{diag}\left\{c_{i}\left(\hat{\xi}_{u}^{\mu}\right)\right\}\right\} \Phi_{u} \Gamma_{u} \bar{q}_{u}^{\mu} d u \\
\approx & \bar{q}_{t_{k-1}}^{\mu}+\Phi_{t_{k}^{\prime}}^{-1} \Gamma_{t_{k}^{\prime}}^{-1} \\
& \times\left\{A+\mu \times \operatorname{diag}\left\{c_{i}\left(\hat{\xi}_{t_{k}^{\prime \prime \prime}}\right)\right\}\right\} \Phi_{t_{k}^{\prime}} \Gamma_{t_{k}^{\prime}} \bar{q}_{t_{k}^{\prime \prime}}^{\mu} \Delta .
\end{aligned}
$$

For the values $\left\{t_{n}^{\prime}, t_{n}^{\prime \prime}, t_{n}^{\prime \prime \prime}\right\}$ in the subinterval $\left(t_{n-1}, t_{n}\right]$ we choose $t_{n}^{\prime}=t_{n}^{\prime \prime}=t_{n}^{\prime \prime \prime}=t_{n-1}$. This choice leads to an explicit approximation scheme

$$
\begin{aligned}
& \bar{q}_{t_{k}}^{\mu} \approx\left[\mathbf{I}+\Delta \Phi_{t_{k-1}}^{-1} \Gamma_{t_{k-1}}^{-1}\{A+\right.\left.\mu \times \operatorname{diag}\left\{c_{i}\left(\hat{\xi}_{t_{k-1}}^{\mu}\right)\right\}\right\} \\
&\left.\times \Phi_{t_{k-1}} \Gamma_{t_{k-1}}\right] \bar{q}_{t_{k-1}} .
\end{aligned}
$$

Finally, multiplying both sides of (3.37) by $\Phi_{t_{k}} \Gamma_{t_{k}}$ we determine a robust version of the risk-sensitive DuncanMortensen-Zakai equation for $q_{t_{k}}^{\mu}$

$$
\begin{aligned}
q_{t_{k}}^{\mu}= & \Phi_{t_{k}} \Phi_{t_{k-1}}^{-1} \Gamma_{t_{k}} \Gamma_{t_{k-1}}^{-1} \\
& \times\left[\boldsymbol{I}+\Delta\left\{A+\mu \times \operatorname{diag}\left\{c_{i}\left(\hat{\xi}_{t_{k-1}}^{\mu}\right)\right\}\right\}\right] q_{t_{k-1}}^{\mu} .
\end{aligned}
$$

Note that the stochastic processes $\left\{y_{t}, 0 \leq t\right\}$ and $\left\{N_{t}, 0 \leq t\right\}$ now appear as parameters in the matrix product $\Phi_{t_{k}} \Phi_{t_{k-1}}^{-1} \Gamma_{t_{k}} \Gamma_{t_{k-1}}^{-1}$ of (3.38) rather than as stochastic integrators in the martingale terms of (3.14).

\section{RISK-SENSITIVE SMOOTHERS}

For smoothed state estimates, we wish to evaluate the expectation $E^{\dagger}\left[X_{t} \mid \mathcal{Y}_{T}\right]$, where $0 \leq t \leq T$.
By a version of Bayes' rule

$$
E\left[X_{t} \mid \mathcal{Y}_{T}\right]=\frac{E^{\dagger}\left[\Lambda_{0, T} X_{t} \mid \mathcal{Y}_{t}\right]}{E^{\dagger}\left[\Lambda_{0, T} \mid \mathcal{Y}_{t}\right]}
$$

The Radon-Nikodym derivative in the right member of (4.39) can be written as a product due to its exponential form, that is, we may write, $\Lambda_{0, T}=\Lambda_{0, t} \Lambda_{t, T}$. This representation proves convenient in establishing smoother dynamics.

Notation: The Radon-Nikodym derivatives $\Lambda_{0, t}$ and $\Lambda_{t, T}$ are defined, respectively, by

$$
\begin{aligned}
\Lambda_{0, t}= & \exp \left(\int_{0}^{t}\left\langle X_{u}, \boldsymbol{g}\right\rangle d u-\frac{1}{2} \int_{0}^{t}\left\langle X_{u}, \boldsymbol{g}\right\rangle^{2} d u\right) \\
& \times \prod_{0<u \leq t}\left\langle X_{u}, \boldsymbol{\lambda}\right\rangle^{\Delta N_{u}} \\
& \times \exp \left(\int_{0}^{t}\left(1-\left\langle X_{u}, \boldsymbol{\lambda}\right\rangle\right) d u\right) \\
\Lambda_{t, T}= & \exp \left(\int_{t}^{T}\left\langle X_{u}, \boldsymbol{g}\right\rangle d u-\frac{1}{2} \int_{t}^{T}\left\langle X_{u}, \boldsymbol{g}\right\rangle^{2} d u\right) \\
& \left.\times \prod_{t<u \leq T}\left\langle X_{u}, \boldsymbol{\lambda}\right\rangle^{\Delta N_{u}}\right) \\
& \times \exp \left(\int_{t}^{T}\left(1-\left\langle X_{u}, \boldsymbol{\lambda}\right\rangle\right) d u\right) .
\end{aligned}
$$

Using the derivatives above and the version of Bayes' rule at (4.39), it is routine to compute robust smoother dynamics. Examples of similar calculations for risk-neutral dynamics are given in [7], . The following theorem is therefore stated without proof.

Theorem 3: Suppose the process $X$ satisfies dynamics given by (2.1) and a vector-valued process $Y$ is observed, as is described by (2.2)-(2.4). For any $t, 0 \leq t \leq T$, the risk-sensitive normalized conditional probability of $X_{t}$, given the observations $\mathcal{Y}_{T}$, is given by

$$
\begin{aligned}
p_{t}^{\mu}=E\left[X_{t} \mid \mathcal{Y}_{T}\right] & =\frac{\sum_{i=1}^{n}\left\langle\bar{q}_{t}^{\mu}, \boldsymbol{e}_{i}\right\rangle\left\langle\bar{v}_{t}^{\mu}, \boldsymbol{e}_{i}\right\rangle \boldsymbol{e}_{i}}{\left\langle\bar{q}_{t}^{\mu}, \bar{v}_{t}^{\mu}\right\rangle} \\
& =\frac{\sum_{i=1}^{n}\left\langle q_{t}^{\mu}, \boldsymbol{e}_{i}\right\rangle\left\langle v_{t}^{\mu}, \boldsymbol{e}_{i}\right\rangle \boldsymbol{e}_{i}}{\left\langle q_{t}^{\mu}, v_{t}^{\mu}\right\rangle} .
\end{aligned}
$$

Here $\bar{q}_{t}^{, \mu}$ satisfies the forward linear ordinary differential equation

$$
\frac{d \bar{q}_{t}^{\mu}}{d t}=\Phi_{t}^{-1} \Gamma_{t}^{-1}\left[A+\mu \times \operatorname{diag}\left\{c_{i}\left(\hat{\xi}_{t}^{\mu}\right)\right\}\right] \Phi_{t} \Gamma_{t} \bar{q}_{t}^{\mu}
$$

and $\bar{v}_{t}^{\mu}$ satisfies the backward linear ordinary differential equation

$$
\frac{d \bar{v}_{t}^{\mu}}{d t}=\Phi_{t}^{-1} \Gamma_{t}^{-1}\left[A^{*}+\mu \times \operatorname{diag}\left\{c_{i}\left(\hat{\xi}_{t}^{\mu}\right)\right\}\right] \Phi_{t} \Gamma_{t} \bar{v}_{t}^{\mu}
$$

The corresponding discrete-time recursions for the processes $q^{\mu}$ and $v^{\mu}$ are, respectively,

$$
\begin{aligned}
q_{t_{k}}^{\mu}= & \Phi_{t_{k}} \Phi_{t_{k-1}}^{-1} \Gamma_{t_{k}} \Gamma_{t_{k-1}}^{-1} \\
& \times\left[\boldsymbol{I}+\Delta\left\{A+\mu \times \operatorname{diag}\left\{c_{i}\left(\hat{\xi}_{t_{k-1}}^{\mu}\right)\right\}\right\}\right] q_{t_{k-1}}^{\mu}
\end{aligned}
$$




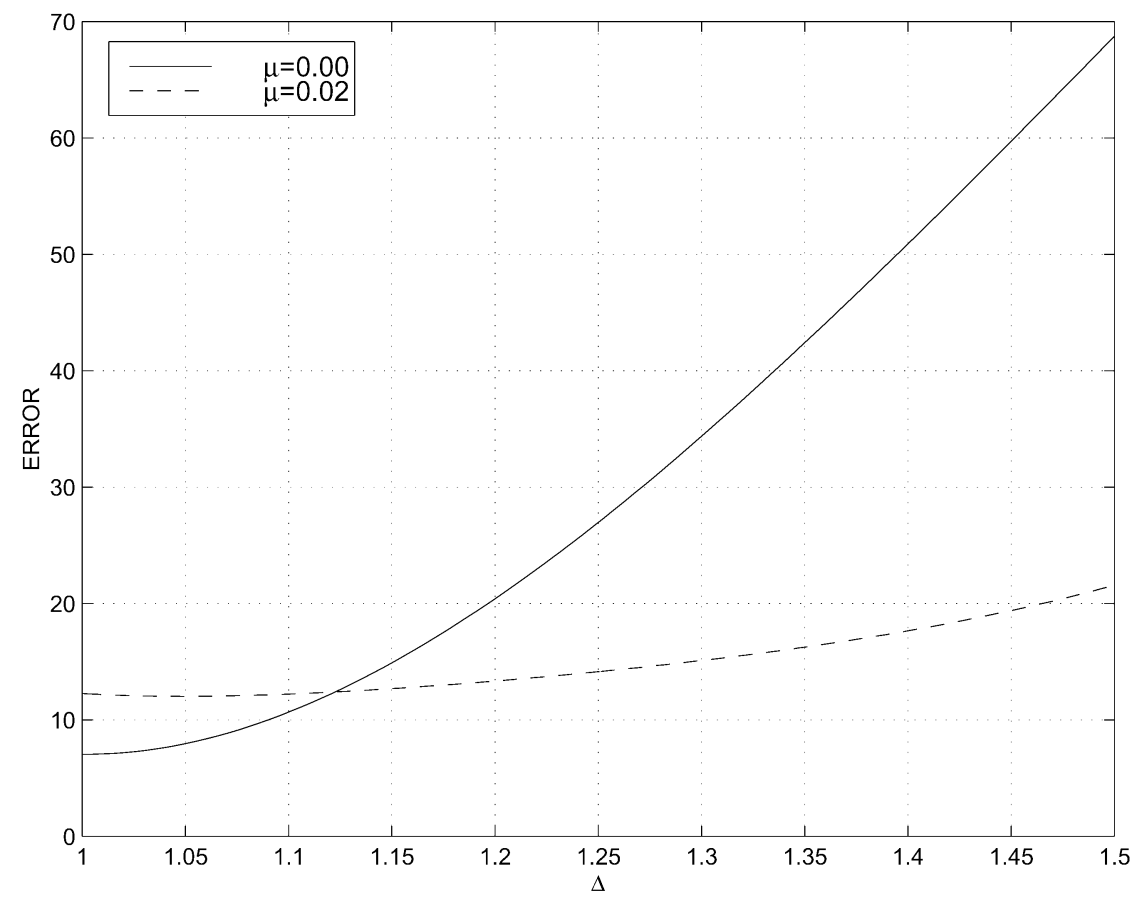

Fig. 1. Estimation error performance comparison for the risk-sensitive and the risk-neutral filters over a family of nominal models. In this study, each of the nominal models is a perturbation of the true model.

and

$$
\begin{aligned}
& v_{t_{k-1}}^{\mu}=\left[\boldsymbol{I}+\Delta\left\{A^{*}+\mu \times\right.\right.\left.\left.\operatorname{diag}\left\{c_{i}\left(\widehat{\xi}_{t_{k-1}}^{\mu}\right)\right\}\right\}\right] \\
& \times \Phi_{t_{k}} \Phi_{t_{k-1}}^{-1} \Gamma_{t_{k}} \Gamma_{t_{k-1}}^{-1} v_{t_{k}}^{\mu}
\end{aligned}
$$

\section{EXAMPLE}

The example we consider here is a Markov process observed through a Poisson process where the stochastic system is the combination of (2.1) and (2.4).

\section{A. Simulation Models}

The True and Fixed Model: The true model used to simulate the observation process $\left\{N_{t}, 0 \leq t\right\}$ is a Poisson process with a jump stochastic rate function defined by

$$
\lambda_{t}=\left\langle X_{t}, \lambda_{0}\right\rangle
$$

where $\lambda_{0}=\{12,27,17,32\}$. For the infinitesimal characteristics of the Markov process $X_{t}$ we define the rate matrix by

$$
A=\left[\begin{array}{cccc}
-a_{1} & \frac{1}{3} a_{1} & \frac{1}{3} a_{1} & \frac{1}{3} a_{1} \\
\frac{1}{3} a_{2} & -a_{2} & \frac{1}{3} a_{2} & \frac{1}{3} a_{2} \\
\frac{1}{3} a_{3} & \frac{1}{3} a_{3} & -a_{3} & \frac{1}{3} a_{3} \\
\frac{1}{3} a_{4} & \frac{1}{3} a_{4} & \frac{1}{3} a_{4} & -a_{4}
\end{array}\right] .
$$

The values taken by the process $\lambda_{t}$ are ascribed expected sojourn times of: $20 \mathrm{~s}$ for the states $\left\{\lambda_{0}^{(1)}, \lambda_{0}^{(3)}\right\}$ and $30 \mathrm{~s}$ for states $\left\{\lambda_{0}^{(2)}, \lambda_{0}^{(4)}\right\}$. These sojourn time statistics correspond to $a_{1}=a_{3}=0.05$ and $a_{2}=a_{4}=0.0333$.
The Design Models: We consider a family of design models where each model is a continuous perturbation of the true model defined by

$$
\lambda_{d}=\{12,27 / \Delta, 17, \Delta 32\}
$$

where the scaling parameter varies in the interval $\Delta \in[1,1.5]$ and $\lambda_{d} \rightarrow \lambda_{0}$ as $\Delta \rightarrow 1$.

\section{B. Results}

Fig. 1 shows the results of a comparison between the risk-sensitive and the risk-neutral filter over a family of design models. At each from a list of the values of $\Delta \in[1,1.5]$, the risk-sensitive and the risk-neutral filters were given an observation realization generated by the true model, yet configured with parameters from a perturbed model according to the value of $\Delta$. The error between the true and the estimated rate functions was calculated by

$$
\text { Error }=1 / T \int_{0}^{T}\left(\lambda_{u}-\widehat{\lambda}_{u}\right)^{2} d u
$$

where $T=3000 \mathrm{~s}$. Note that this is a time integral of the squared error, not an expectation of the squared error.

Error performance curves such as those in Fig. 1 provide an illustration of the possible benefits of risk-sensitive filtering. The robust filter used to generate the curve in Fig. 1 demonstrates an estimation error which degrades more gracefully that the corresponding risk-neutral filter. The comparison of the curves in Fig. 1 shows the a risk-sensitive filter can offer robustness against model uncertainties.

In Fig. 2, we show the error defined by (5.51) calculated as a function of the parameter $\mu$ for a fixed value of $\Delta=1.5$. The minimum of this curve marks the best risk-sensitive parameter for this specific scenario. In Fig. 3, we show a family of error 


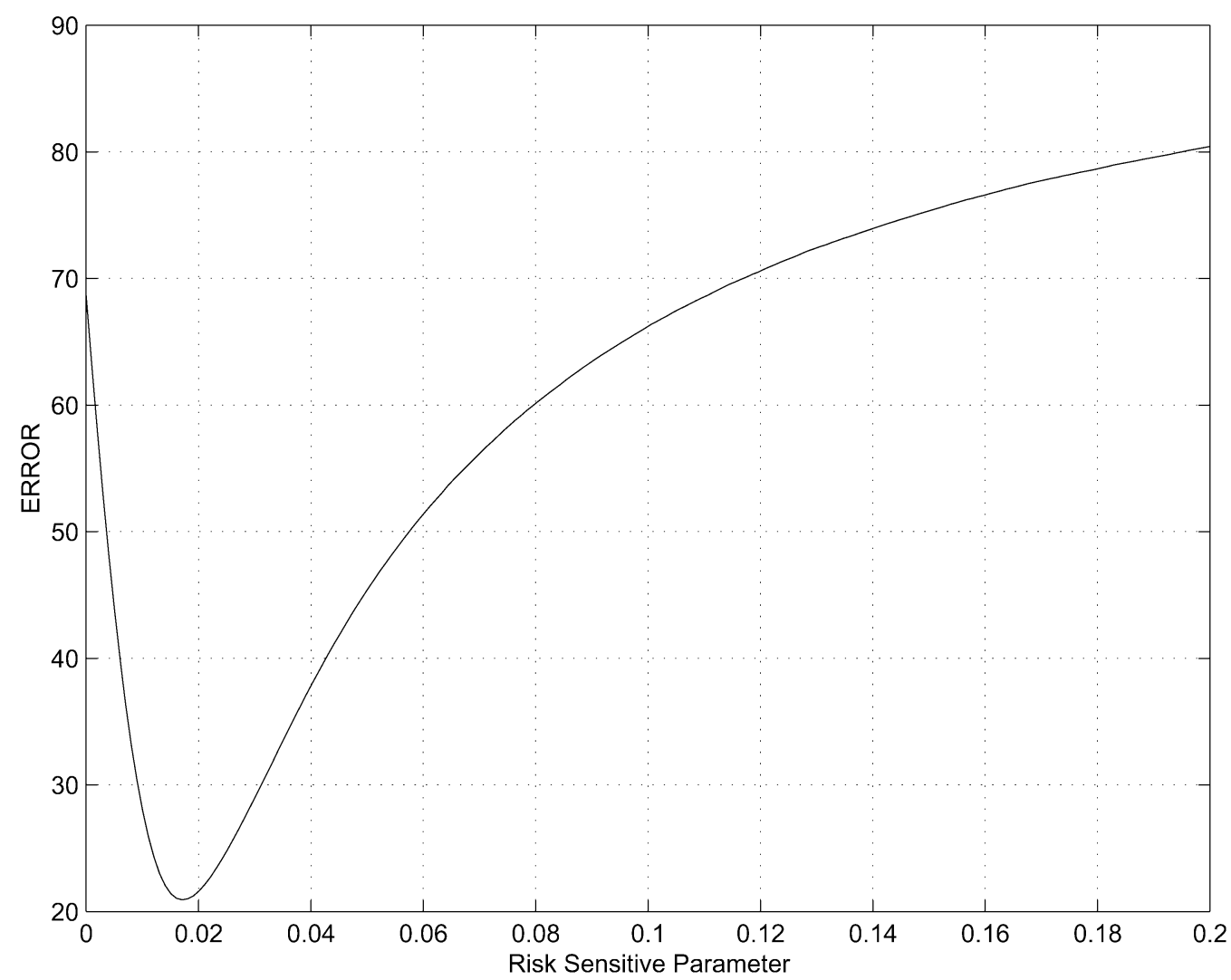

Fig. 2. Estimation error for the risk-sensitive filter as a function of the risk-sensitive parameter $\mu$ in the scenario $\Delta=1.5$.

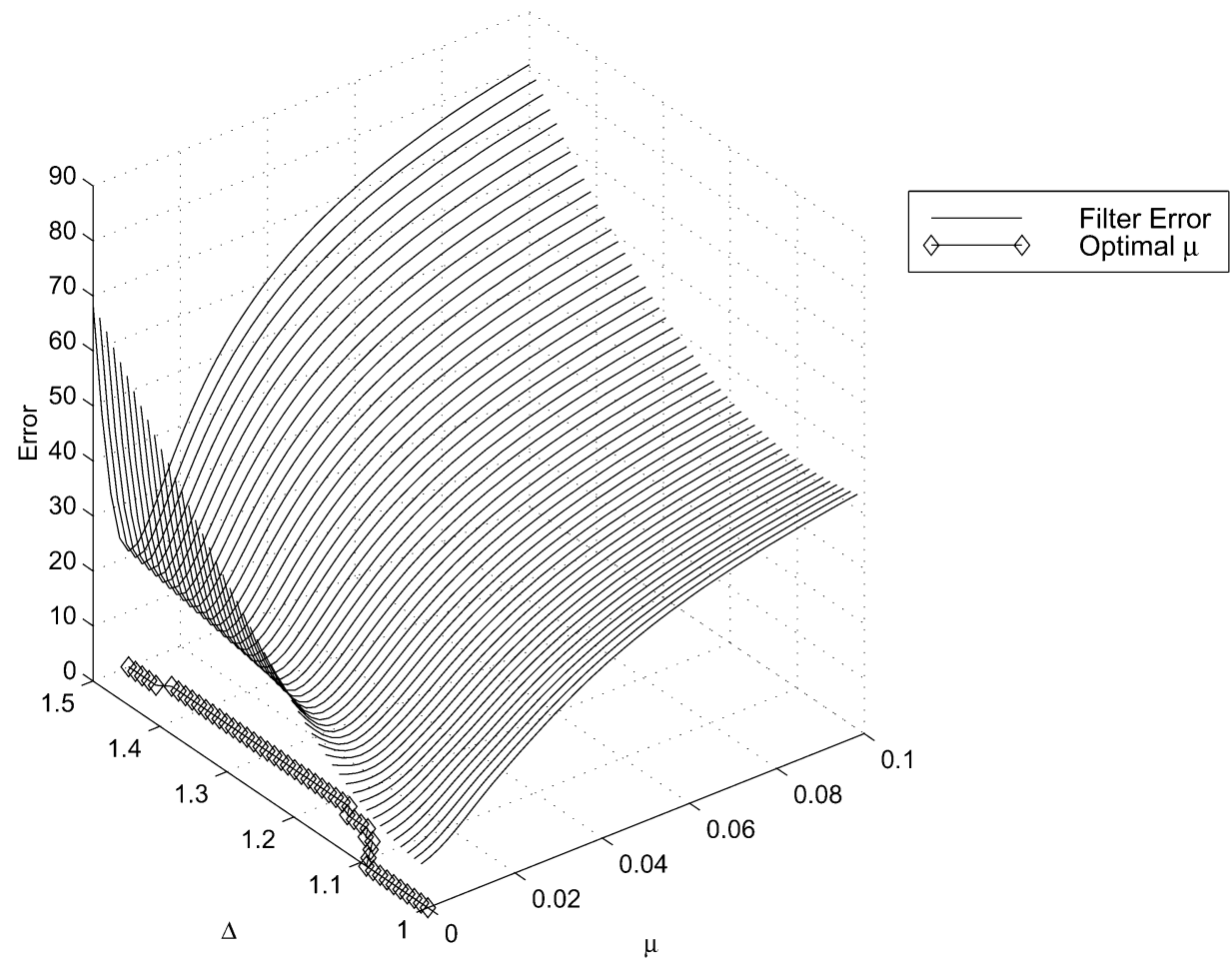

Fig. 3. Risk-sensitive filter error curves as a function of both the parameter $\mu$ and the number $\Delta$. Here the number $\Delta$ quantifies the degree of difference between the true and design probability models. $\Delta=1$ corresponds to a scenario where the true and design probability models coincide. 


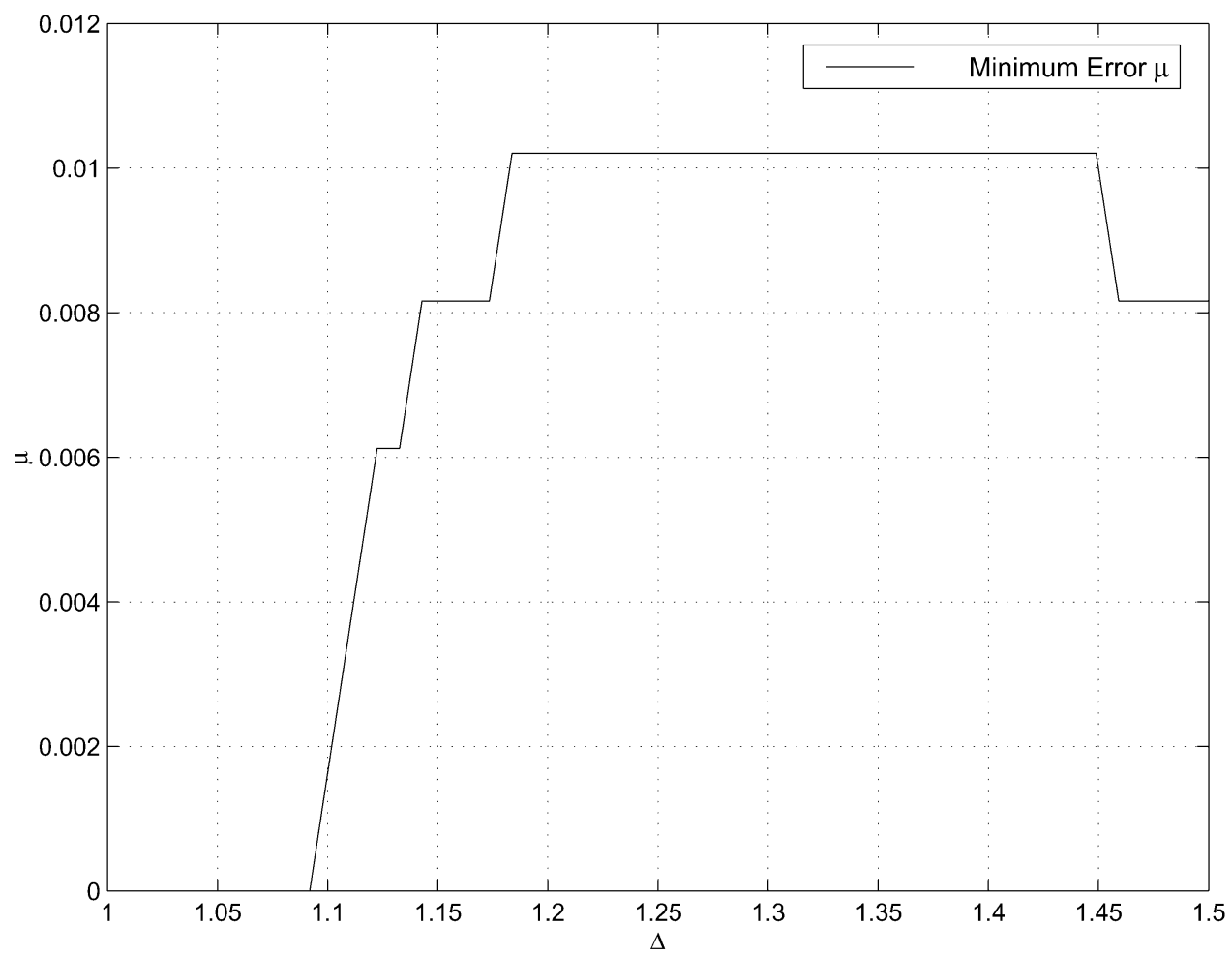

Fig. 4. The optimal value $\mu$ as a function of the number $\Delta$. This curve shows the value of $\mu$ that is required to achieve the best performance for a given scenario depending upon $\Delta$.

curves such as the single curve shown in Fig. 2. Each curve in Fig. 3 represents a particular scenario, where the degree of difference between the true and design probability models is determined by the number $\Delta$. In simulation studies to generate families of curves such as those in Fig. 3, it was observed that the local curvature about the minima changed with $\Delta$, such that choosing a "good" value for $\mu$ became increasingly difficult as the true and design models became more distinct. It should be further noted, that although the curves in Fig. 3 suggest the existence and uniqueness of optimal values for $\mu$, these curves were determined via complete knowledge of the true Markov state process. Perceivably, one could estimate the location of the optimal $\mu$ if an equation for the state estimator error variance were available. In Fig. 4, the optimal $\mu$ (risk-sensitive parameter corresponding to the minima of the curves in Fig. 3) is plotted for values of $\Delta$ shown in Fig. 3.

\section{CONCLUSION}

In this paper, we presented risk-sensitive filters and smoothers for an observation process whose components represent the observation of Markov process through a Brownian motion and the observation of a Markov process through a Poisson process. Using a gauge transformation, a robust (to discretization) version of the Duncan-Mortensen-Zakai equation corresponding to our risk-sensitive filter was obtained. A simulation study for an example of counting process observations was provided.

\section{A. Proof of Theorem 1}

The proof of this Theorem has two parts. In the first part, we use the Ito product rule to determine the semimartingale $\Lambda_{t} X_{t} Z_{t}$. In the second part, we condition this semimartingale under the reference probability $P^{\dagger}$.

The semimartingale $\Lambda_{t}$ satisfies the stochastic integral equation (see [8])

$\Lambda_{t}=1+\int_{0}^{t} \Lambda_{u}\left\langle X_{u}, g\right\rangle d y_{u}+\int_{0}^{t} \Lambda_{u-}\left(\left\langle X_{s}, \lambda\right\rangle-1\right)\left(d N_{u}-d u\right)$

First we note that

$$
\begin{aligned}
d \Lambda_{t}= & \Lambda_{t}\left\langle X_{t}, \boldsymbol{g}\right\rangle d y_{t} \\
& +\Lambda_{t-}\left(\left\langle X_{t}, \boldsymbol{\lambda}\right\rangle-1\right)\left(d N_{t}-d t\right), \\
d Z_{t}= & \mu c\left(\xi_{t}, \hat{\xi}_{t}^{\mu}\right) Z_{t} d t, \\
d X_{t}= & A X_{t} d t+d M_{t},
\end{aligned}
$$

then

$$
\begin{aligned}
\Lambda_{t} X_{t} Z_{t}= & X_{0}+\int_{0}^{t} \Lambda_{u} A X_{u} Z_{u} d u+\int_{0}^{t} \Lambda_{u} Z_{u} d M_{u} \\
& +\int_{0}^{t} \Lambda_{u} X_{u} Z_{u}\left\langle X_{u}, \boldsymbol{g}\right\rangle d y_{u} \\
& +\int_{0}^{t} \Lambda_{u-} X_{u} Z_{u}\left(\left\langle X_{u}, \lambda\right\rangle-1\right)\left(d N_{u}-d u\right) \\
& +\int_{0}^{t} \mu \Lambda_{u} X_{u} Z_{u} c\left(\xi_{u}, \hat{\xi}_{u}^{\mu}\right), d u .
\end{aligned}
$$


By using Fubini's theorem ([8]) on (A56), noting that under the reference measure $P^{\dagger}$, both $y_{t}$ and $N_{t}$ have independent increments, and

$$
E^{\dagger}\left[\int_{0}^{t} Z_{u} \Lambda_{u}\left\langle d M_{u}, X_{u}\right\rangle \mid \mathcal{Y}_{t}\right]=0
$$

then the result follows.

\section{B. Proof of Corollary 1}

Proof: Note that $E^{\dagger}\left[\Lambda_{t} \mid \mathcal{Y}_{t}\right]=\left\langle q_{t}^{0}, 1\right\rangle=\widehat{\Lambda}_{t}$. To prove Corollary 1, we follow the method in [9] in which one uses the special semimartingale $\widehat{\Lambda}_{t}^{-1}$ and the Ito rule to write down the the product $q_{t}^{\mu} \widehat{\Lambda}_{t}^{-1}$. This, by definition is the process $\Pi_{t}^{\mu}$. The semimartingale decomposition for the process $\widehat{\Lambda}_{t}$ reads [8]

$$
\begin{aligned}
\widehat{\Lambda}_{t}=1+\int_{0}^{t} & \widehat{\Lambda}_{u-}\left\langle\Pi_{u}^{0}, g\right\rangle d y_{u} \\
& +\int_{0}^{t} \widehat{\Lambda}_{u-}\left(\left\langle\Pi_{u-}^{0}, \boldsymbol{\lambda}\right\rangle-1\right)\left(d N_{u}-d u\right) .
\end{aligned}
$$

Note that the terms $\widehat{\Lambda}_{t},\left\langle\Pi_{t}^{0}, \boldsymbol{g}\right\rangle$, and $\left\langle\Pi_{t}^{0}, \boldsymbol{\lambda}\right\rangle$ in (A58) are projections under different probability measures: where

$$
\begin{gathered}
\widehat{\Lambda}_{t} \triangleq E^{\dagger}\left[\Lambda_{t} \mid \mathcal{Y}_{t}\right], \\
\Pi_{t}^{0} \triangleq E\left[X_{t} \mid \mathcal{Y}_{t}\right] .
\end{gathered}
$$

Now

$$
\begin{gathered}
\widehat{\Lambda}_{t}^{-1}=1-\int_{0}^{t} \widehat{\Lambda}_{u-}^{-1}\left\langle\Pi_{u}^{0}, \boldsymbol{g}\right\rangle^{2} d u-\int_{0}^{t} \widehat{\Lambda}_{u-}^{-1}\left\langle\Pi_{u}^{0}, \boldsymbol{g}\right\rangle d y_{u} \\
-\int_{0}^{t} \hat{\Lambda}_{u-}^{-1}\left\{\frac{\left\langle\Pi_{u}^{0}, \boldsymbol{\lambda}\right\rangle-1}{\left\langle\Pi_{u}^{0}, \boldsymbol{\lambda}\right\rangle}\right\}\left(d N_{u}-\left\langle\Pi_{u}^{0}, \lambda\right\rangle d u\right) .
\end{gathered}
$$

Therefore,

$$
\begin{aligned}
d\left(q_{t}^{\mu} \widehat{\Lambda}_{t}^{-1}\right)=\widehat{\Lambda}_{t}^{-1} d q_{t}^{\mu}+q_{t}^{\mu} d \widehat{\Lambda}_{t}^{-1}+d\left[q_{t}^{\mu} \widehat{\Lambda}_{t}^{-1}\right] & \\
=\widehat{\Lambda}_{t}^{-1}\{ & {\left[A+\mu \times \operatorname{diag}\left\{c_{i}\left(\hat{\xi}_{t}^{\mu}\right)\right\}\right] q_{t}^{\mu} d t } \\
& +\operatorname{diag}\left\{\left\langle\boldsymbol{g}, \boldsymbol{e}_{i}\right\rangle\right\} q_{t}^{\mu} d y_{t} \\
& \left.\times \operatorname{diag}\left\{\left\langle\boldsymbol{\lambda}, \boldsymbol{e}_{i}\right\rangle-1\right\} q_{t-}^{\mu} d Q_{t}\right\} \\
+q_{t}^{\mu}\{ & -\widehat{\Lambda}_{t}^{-1}\left\langle\Pi_{t}^{0}, \boldsymbol{g}\right\rangle^{2} d t-\widehat{\Lambda}_{t-}^{-1}\left\langle\Pi_{t}^{0}, \boldsymbol{g}\right\rangle d y_{t} \\
& -\widehat{\Lambda}_{t-}^{-1}\left\{\frac{\left\langle\Pi_{t-}^{0}, \boldsymbol{\lambda}\right\rangle-1}{\left\langle\Pi_{t-}^{0}, \boldsymbol{\lambda}\right\rangle}\right\} \\
& \left.\times\left(d N_{t}-\left\langle\Pi_{t-}^{0}, \boldsymbol{\lambda}\right\rangle d t\right)\right\}
\end{aligned}
$$

$$
\begin{aligned}
& -\operatorname{diag}\left\{\left\langle\boldsymbol{\lambda}, \boldsymbol{e}_{i}\right\rangle-1\right\} q_{t-}^{\mu} \widehat{\Lambda}_{t-}^{-1} \\
& \times\left\{\frac{\left\langle\Pi_{t-}^{0}, \boldsymbol{\lambda}\right\rangle-1}{\left\langle\Pi_{t-}^{0}, \boldsymbol{\lambda}\right\rangle}\right\} d N_{t} \\
& \left.-\widehat{\Lambda}_{t}^{-1}\left\langle\Pi_{t}^{0}, \boldsymbol{g}\right\rangle q_{t-}^{\mu} \operatorname{diag}\left\{\left\langle\boldsymbol{g}, \boldsymbol{e}_{i},\right\rangle\right\rangle\right\} d t .
\end{aligned}
$$

Writing $\widehat{\Lambda}_{t}^{-1} q_{t}^{\mu}=\Pi_{t}^{\mu}$ we see

$$
\begin{aligned}
d\left(q_{t}^{\mu} \widehat{\Lambda}_{t}^{-1}\right)= & {\left[A+\mu \times \operatorname{diag}\left\{c_{i}\left(\hat{\xi}_{t}^{\mu}\right)\right\}\right] \Pi_{t}^{\mu} d t } \\
& +\operatorname{diag}\left\{\left\langle\boldsymbol{g}, \boldsymbol{e}_{i}\right\rangle\right\} \Pi_{t}^{\mu} d y_{t} \\
& -\Pi_{t}^{\mu}\left\langle\Pi_{t}^{0}, \boldsymbol{g}\right\rangle d y_{t} \\
& +\Pi_{t}^{\mu}\left\langle\Pi_{t}^{0}, \boldsymbol{g}\right\rangle^{2} d t \\
& -\operatorname{diag}\left\{\left\langle\boldsymbol{g}, \boldsymbol{e}_{i}\right\rangle\right\} \Pi_{t}^{\mu}\left\langle\Pi_{t}^{0}, \boldsymbol{g}\right\rangle d t \\
& +\operatorname{diag}\left\{\left\langle\boldsymbol{\lambda}, \boldsymbol{e}_{i}\right\rangle-1\right\} \Pi_{t-}^{\mu}\left(d N_{t}-d t\right) \\
& -\Pi_{t-}^{\mu}\left\{\frac{\left\langle\Pi_{t-}^{0}, \boldsymbol{\lambda}\right\rangle-1}{\left\langle\Pi_{t-}^{0}, \boldsymbol{\lambda}\right\rangle}\right\}\left(d N_{t}-\left\langle\Pi_{t}^{0}, \boldsymbol{\lambda}\right\rangle d t\right) \\
& -\operatorname{diag}\left\{\left\langle\boldsymbol{\lambda}, \boldsymbol{e}_{i}\right\rangle-1\right\} \\
& \times \Pi_{t-}^{\mu}\left\{\frac{\left\langle\Pi_{t-}^{0}, \boldsymbol{\lambda}\right\rangle-1}{\left\langle\Pi_{t-}^{0}, \boldsymbol{\lambda}\right\rangle}\right\} d N_{t} .
\end{aligned}
$$

With simple rearrangement, Corollary 1 follows.

\section{ACKNOWLEDGMENT}

W. P. Malcolm would like to gratefully acknowledge Dr. Daryl J. Daley for many helpful technical discussions concerning doubly stochastic Poisson processes.

\section{REFERENCES}

[1] R. Boel, M. R. James, and I. R. Peterson, "Robustness and risk sensitive filtering," IEEE Trans. Automat. Control, vol. 47, no. 3, pp. 265-271, Mar. 2002.

[2] D. R. Shin and E. I. Verriest, "The general formulas for smoothing and prediction of mixed-type observations," in Proc. IEEE Conf. Decision and Control, Honolulu, HI, Dec. 1990, pp. 810-814.

[3] J. M. C. Clark, "The design of robust approximations to the stochastic differential equations for nonlinear filtering," in Communications Systems and Random Process Theory, J. K. Skwirzynski, Ed. Alphen aan den Rijn, The Netherlands: Sijthoff and Noorhoff, 1978, pp. 721-734.

[4] R. J. Elliott, L. Aggoun, and J. B. Moore, Hidden Markov Models Estimation and Control, ser. Applications of Mathematics. Berlin, Germany: Springer-Verlag, 1995.

[5] P. Whittle, Risk Sensitive Optimal Control. New York: Wiley, 1990.

[6] P. J. Huber, Robust Statistical Procedures, ser. CBMS-NSF Regional Conference Series In Applied Mathematics. Philadelphia, PA: SIAM, 1996.

[7] W. P. Malcolm and R. J. Elliott, "A general smoothing equation for Poisson observations," in Proc. IEEE Conf. Decision and Control, Phoenix, AZ, Dec. 1999, pp. 4106-4110.

[8] E. Wong and B. Hajek, Stochastic Processes in Engineering Systems. New York: Springer-Verlag, 1985.

[9] R. J. Elliott, Stochastic Calculus and its Applications. Berlin, Germany: Springer-Verlag, 1982. 